\title{
Regulación de la liberación de renina durante la hipertensión renovascular
}

\author{
Elizabeth Alejandrina Guzmán-Hernández ${ }^{1}$, David Segura-Cobos ${ }^{2}$, Maximiliano Ibarra-Barajas ${ }^{1}$ \\ ${ }^{1}$ Unidad de Biomedicina. ${ }^{2}$ Laboratorio de Farmacología, Unidad de Investigación Interdisciplinaria de Ciencias de la \\ Salud y la Educación, Facultad de Estudios Superiores Iztacala, Universidad Nacional Autónoma de México, Tlalnepantla, \\ Estado de México, México
}

\section{RESUMEN}

La hipertensión es un síndrome cardiovascular progresivo que surge de etiologías complejas e interrelacionadas. Los marcadores tempranos del síndrome a menudo están presentes antes de que la elevación de la presión sanguínea sea observada. El desarrollo de la hipertensión arterial se asocia con anomalías cardíacas y vasculares funcionales y estructurales que dañan el corazón, los riñones, el cerebro, los vasos, y otros órganos, y conducen a la morbilidad y muerte prematura. El sistema renina angiotensina y los riñones son los principales mecanismos que subyacen para el desarrollo de la hipertensión. La renina es la enzima limitante para la síntesis de la angiotensina II; la liberación de renina está regulada por mecanismos como el barorreceptor intrarrenal, la mácula densa (MD) y el sistema nervioso simpático. Desde la MD son liberadas prostaglandinas vasodilatadoras $(\mathrm{PG})$ como $\mathrm{PGI}_{2}$ y $\mathrm{PGE}_{2}$, generadas por la ciclooxigenasa 2, que inducen la liberación de renina de las células yuxtaglomerulares. En esta revisión, mostramos los mecanismos interrelacionados entre la ciclooxigenasa 2 de la MD y la angiotensina II renal.

Palabras clave: renina, ciclooxigenasa 2, hipertensión arterial.

\section{ABSTRACT \\ Regulation of renin release in renovascular hypertension}

Hypertension is a progressive cardiovascular syndrome arising from complex and interrelated etiologies. Early markers of the syndrome are often present before blood pressure elevation is observed. The development of arterial hypertension is associated with functional and structural cardiac and vascular abnormalities that damage the heart, kidneys, brain, vasculature, and other organs, and lead to morbidity and premature death. The kidney and the renin angiotensin system are the principal mechanisms that underlie for development of hypertension. Renin is the rate limiting enzyme for angiotensin II synthesis, and renin release is regulated by mechanisms as the intrarenal baroreceptor, the macula densa (MD) and the sympathetic nervous system. The MD releases vasodilator prostaglandins (PG) as PGI2 and PGE2, generated by cyclooxygenase 2 , which induce renin release from juxtaglomerular cells. In this review, we show interrelated mechanisms between cyclooxygenase 2 of $\mathrm{MD}$ and renal angiotensin II.

Key words: renin, cyclooxygenase 2, arterial hypertension

\footnotetext{
Autor para correspondencia: Elizabeth Alejandrina Guzmán-Hernández, Unidad de Biomedicina, Facultad de Estudios Superiores Iztacala, Universidad Nacional Autónoma de México, Avenida de los barrios 1, Colonia Reyes-Iztacala, Tlalnepantla, Estado de México, C.P. 54090, México . E-mail: shponia2000@yahoo.com.mx
}

Recibido: el 1 de agosto de 2014 Aceptado para publicación: el 15 de diciembre de 2014

Este documento está disponible en http://www.revbiomed.uady.mx/pdf/rb152615.pdf

Vol. 26, No. 1, enero-abril de 2015 
Guzmán-Hernández et al.

\section{INTRODUCCIÓN}

La hipertensión arterial (HTA) es una de las enfermedades crónicas más comunes de la humanidad que afecta a más de mil millones de personas en todo el mundo (1). De acuerdo a la Encuesta Nacional de Salud 2012, en México, se estima que 1 de cada 3 mexicanos adultos padece HTA; la mayor prevalencia se observa en mujeres a partir de los 50 años (2).

Aunque la presión arterial elevada por sí misma no causa, típicamente, síntomas evidentes, las consecuencias de la hipertensión crónica incluyendo la hipertrofia cardiaca, insuficiencia cardíaca, accidente cerebrovascular y enfermedad renal, son responsables de una elevada morbilidad y mortalidad (3).

Se ha propuesto que el riñón y el sistema renina angiotensina, son los principales mecanismos que subyacen al desarrollo de la HTA. Evidencia creciente muestra la relevancia del riñón en la regulación a largo plazo de la presión arterial mediante el control del volumen del fluido extracelular (4).

El equilibrio se consigue, normalmente, haciendo coincidir la excreción urinaria de sal y agua con la ingesta alimentaria, manteniendo así un volumen de líquido extracelular y de presión arterial constantes. Guyton, llamó a este efecto natriuresis por presión, donde el riñón debe reajustar su relación presión normal a expensas de mayor contracción de las arteriolas eferentes renales, disminuir el flujo sanguíneo renal, aumentar la fracción de filtración e incrementar la retención de $\mathrm{Na}^{+}$; con aumento del volumen extracelular en exceso, para mantener normal la presión arterial; al incrementar el volumen circulatorio, la resistencia arteriolar se mantiene elevada, por lo que hay mayor esfuerzo cardiaco para impulsar la sangre hacia las arterias, que son sometidas a gran presión, ocasionando la pérdida de la elasticidad, y desarrollo de la HTA (5).

A través de los años, una serie de estudios de trasplante de riñón han apoyado un papel clave para las funciones intrínsecas del riñón en la patogenia de la HTA. Por ejemplo, el trasplante de un riñón de una rata hipertensa Dahl a una rata normotensa, desarrolla HTA, independientemente de que se realice el trasplante antes o después de una dieta alta en sal. Por el contrario, el trasplante de un riñón de un animal normotenso resistente a la sal a una rata sensible a la sal no desarrolla HTA. En los seres humanos, también se ha observado este efecto, pacientes con HTA que reciben un riñón de un paciente normotenso no desarrollan HTA. En conjunto, estos estudios indican que un defecto en la excreción de sodio por el riñón confiere susceptibilidad a la presión arterial elevada (6).

\section{Acciones intrarrenales del sistema renina} angiotensina en el control de la presión arterial. El sistema renina angiotensina aldosterona (SRA) es uno de los mecanismos más importantes en la regulación de la presión arterial y en la hemodinámica renal; su desregulación promueve la retención de sodio y agua, con el consecuente desarrollo de la HTA. El bloqueo farmacológico del SRA con inhibidores de la renina o de la enzima convertidora de angiotensina (IECA) o antagonistas de los receptores de la angiotensina reduce eficazmente la presión arterial en la mayoría de los pacientes con HTA, lo que refleja el papel importante del SRA en el desarrollo de la HTA en los seres humanos (7).

La participación de la angiotensina II (Ang II) en el riñón para influir en la regulación de la presión arterial está bien establecida. La infusión de dosis bajas de Ang II de manera sostenida induce el desarrollo de la HTA con alteración en la natriuresis por presión (8). Navar y col. (2011), mostraron la existencia del control local e independiente de la actividad del SRA dentro del riñón que influye en la excreción de sodio y en la regulación de la presión arterial. En consecuencia, la concentración elevada de Ang II circulante se asocia con la acumulación de péptidos de angiotensina en el riñón, al aumento en la expresión del angiotensinógeno

\section{Revista Biomédica}




\section{Regulación de renina en hipertensión renovascular}

en el epitelio del túbulo proximal y la excreción de angiotensinógeno.

En el riñón, la Ang II a través de su unión al receptor AT1 influye en la actividad de varios transportadores epiteliales de sodio como el cotrasportador $\mathrm{Na}^{+} / \mathrm{H}^{+}$y el $\mathrm{Na}^{+} / \mathrm{HCO}^{-}$en túbulo proximal, el canal de sodio sensible a amilorida (ENAC) y el cotrasportador $\mathrm{Na}^{+} / \mathrm{Cl}^{-}$en la nefrona distal (9).

Matsusaka e Ichikawa (2012), utilizando ratones con deleción específica para el gen del angiotensinógeno de riñón o hígado, mostraron que la mayor parte del angiotensinógeno y de Ang II en el riñón principalmente, se deriva del angiotensinógeno producido por el hígado (10). Por otra parte, la deleción genética del angiotensinógeno en el riñón, redujo significativamente la excreción de angiotensinógeno en la orina, consistente con los estudios de Navar y col. (1999), quienes mostraron que la Ang II actúa sobre el lumen de la nefrona y es el componente efector crítico de la activación intrarrenal del SRA (11).

Estudios realizados por Crowley y col (2011), mostraron que la HTA se manifiesta por la presencia de los receptores AT1 renales (12). Otros estudios han mostrado que la deleción del receptor AT1 en el epitelio tubular proximal, confiere un efecto protector para el desarrollo de la HTA dependiente de Ang II, ya que se reduce la activación de los transportadores de sodio (13), mientras que, en el conducto colector Ang II estimula directamente la actividad del canal epitelial de sodio $(\mathrm{ENaC})$, promoviendo de esta manera la retención de sodio y agua (14). Evidencia creciente muestra la existencia de una interrelación entre la COX-2 derivada de la mácula densa (MD) y los componentes del SRA (15).

Metabolismo del ácido araquidónico. El ácido araquidónico (AA) es un ácido graso poliinsaturado, constituido por 20 átomos de carbono, cuya oxidación origina mediadores lipídicos que reciben el nombre de eicosanoides. En los mamíferos, en respuesta a diversos estímulos que dependen del tipo celular, se activan diferentes receptores que activan a su vez varias fosfolipasas (PL), destacando la PLA2 citosólica como la principal involucrada en la liberación del AA de las membranas celulares (16).

La ciclooxigenasa (COX), o prostaglandina endoperóxido sintasa, es una proteína de membrana que cataliza la formación de diversos prostanoides a partir del AA, y es inhibida por los antiinflamatorios no esteroideos (AINEs). En la actualidad se conocen dos isoformas de la COX, la COX-1 y la COX-2, que presentan un peso molecular muy similar (de $70 \mathrm{kDa}$ ); a pesar de que sus proteínas no son tan semejantes, conservan un $60 \%$ de homología en sus aminoácidos (16).

El primer paso hacia la formación de prostaglandinas (PGs) es la liberación del AA de los fosfolípidos de la membrana, normalmente, por la acción de la PLA2 citosólica. Una vez liberado el AA, tanto COX-1 como COX-2, lo transforman en $\mathrm{PGG}_{2}$ y $\mathrm{PGH}_{2}$ por idénticas reacciones; de hecho, la $\mathrm{COX}$ es una enzima dual que adiciona dos moléculas de oxígeno al AA para formar $\mathrm{PGG}_{2} \mathrm{y}$, después, reduce a este hidroperóxido cíclico en el endoperóxido altamente activo $\mathrm{PGH}_{2}$ que actúa como sustrato intermedio para la síntesis de PGs de las series E2, F2, D2, e I2 y el tromboxano A2 (TXA2). Debido a su rápida degradación, las acciones biológicas de las PGs se producen de manera autocrina y paracrina (16).

Regulación de la expresión de la ciclooxigenasa-2 en la mácula densa. La mácula densa (MD) es una región especializada de 15-20 células, formada por una membrana basal cubierta por pequeñas prolongaciones llamados cilios que se encuentran en contacto con la velocidad del fluido luminal, que llega a la MD y una membrana apical en donde se localiza el cotrasportador $\mathrm{Na}^{+} / \mathrm{K}^{+} / 2 \mathrm{Cl}^{-}(\mathrm{NKCC} 2)$ cuya función es la de sensar la concentración de $\mathrm{NaCl}$ que llega a la MD (17). 


\section{Guzmán-Hernández et al.}

E1 NKCC2 tiene una gran afinidad por el $\mathrm{Na}^{+} \mathrm{y} \mathrm{K}^{+}$, y menor afinidad para el Cl- con lo cual resulta en un mecanismo de absorción que es muy sensible a cualquier cambio en el cloruro luminal (18).

En la MD se localiza la COX-2, la cual participa en la regulación de la liberación de renina y en el mantenimiento del tono arteriolar aferente. Se ha observado que bajo ciertas condiciones aumenta la expresión y la actividad de la COX-2 y de renina de manera simultánea, dentro de estas condiciones se encuentran: La administración de una dieta baja en sal, la inhibición de la enzima convertidora de angiotensina, la administración de diuréticos y la hipertensión renovascular (19).

En ratas hipertensas por coartación aórtica, que es un modelo de HTA cuya característica principal es la disminución renal hacia uno de los riñones, se ha observado que tras la reducción en el flujo sanguíneo aumenta la expresión de la COX-2 y de manera paralela la expresión de renina (19), por lo que al administrar inhibidores selectivos de la COX-2, como el SC-58236 o celecoxib, la actividad de renina plasmática se reduce, y con ello disminuye la actividad del sistema renina angiotensina indicando que, una de las vías de estimulación renal del sistema renina angiotensina es a través de las prostaglandinas derivadas de la COX-2 (20). La administración de diuréticos de asa, que inhiben la actividad del cotransportador NKCC2, aumentan la expresión y la actividad de la COX-2 y de renina, incluso en la ausencia de depleción de volumen (21). Estudios realizados en cultivos celulares de la porción ascendente gruesa del asa de Henle mostraron que la expresión de la COX-2 en la MD aumenta en respuesta a la disminución de cloruro extracelular (22). Estudios realizados utilizando diferentes diuréticos que inhiben algunos sistemas de transporte en la nefrona, mostraron que la acetazolamida que inhibe la actividad de la anhidrasa carbónica en el túbulo proximal, incrementa la concentración de $\mathrm{NaCl}$ que llega a la MD, el cual es sensado por el cotrasportador
NKCC2 como un aumento en la recaptura de $\mathrm{Na}^{+}$ y $\mathrm{Cl}^{-}$hacia el interior de la $\mathrm{MD}$, por lo que se inhibe la expresión de la COX-2 y la liberación de renina; mientras que, la hidroclorotiazida, la amilorida, la furosemida y la espironolactona, inhiben la reabsorción en el túbulo contorneado distal, por lo que la concentración de $\mathrm{Na}^{+} \mathrm{y} \mathrm{Cl}^{-}$ que llega a la MD es menor y es sensado por el cotrasportador NKCC2 como una caída en la concentración de estos iones (23), por lo que se induce la fosforilación de la MAP cinasa p38 y ERK1/2 y, a través del factor nuclear $\kappa \mathrm{B}(\mathrm{NF} \kappa \mathrm{B})$, se induce la expresión de COX-2 (23).

Interacción de la ciclooxigenasa-2 con el sistema renina angiotensina. La renina es la enzima limitante para la formación de Ang II, se libera de las células yuxtaglomerulares que se encuentran en el aparato yuxtaglomerular, el cual es una región de contacto especializado entre el glomérulo y la porción ascendente gruesa del asa de Henle cortical, desempeña un papel activo en el mantenimiento de la homeostasis iónica, en el control de la presión arterial y en la liberación de renina (24). El aparato yuxtaglomerular consta de un componente tubular, la mácula densa, y un componente vascular que consiste en las arteriolas aferentes y eferentes, así como de un mesangio extraglomerular.

El aparato yuxtaglomerular se encuentra formado por varios tipos de células como las células del músculo liso vascular, células endoteliales, células mesangiales, células de la MD, y células granulares yuxtaglomerulares secretoras de renina (24) (Figura 1).

Otros estudios han mostrado expresión de renina en otras regiones de la nefrona como el conducto colector (25). Sin embargo, la mayor producción de esta enzima se lleva a cabo en las células yuxtaglomerulares donde es regulada por el barorreceptor intrarrenal, la mácula densa y el sistema nervioso simpático (26).

Barorreceptor intrarrenal. El barorreceptor se encuentra localizado en la vasculatura renal, 
Regulación de renina en hipertensión renovascular

específicamente en la arteriola aferente y su función es sensar los cambios en la presión sanguínea (27). En perros concientes mostraron que el barorreceptor intrarrenal se activa cuando disminuye la presión sanguínea de 100 a 60 $\mathrm{mmHg}$, lo que estimula la liberación de renina hasta 7 veces, mientras que el incremento de la presión sanguínea de 100 a $140 \mathrm{mmHg}$ inhibe la liberación de renina (Figura 1).

Vía del receptor $\beta$-adrenérgico. Los efectos sobre la secreción y liberación de renina son mediados por la activación de los receptores adrenérgicos $\beta 1 / \beta 2$, lo cuales se expresan en las terminales nerviosas pre- $\mathrm{y}$ posganglionares ubicadas en diversas regiones del riñón incluyendo el polo vascular glomerular donde se entremezclan con las células yuxtaglomerulares. La deleción de estos receptores reduce significativamente la expresión de renina; esta reducción es mayor que la observada solo por el bloqueo farmacológico de los receptores $\beta$-adrenérgicos o en riñones denervados (28).

Mácula densa. La mácula densa (MD) participa en la liberación de renina a través de la COX-2 y de la síntesis de prostanoides vasodilatadores. Estas observaciones son apoyadas a partir de estudios realizados in vitro en aparato yuxtaglomerular aislado de conejo a los que se les administraron de manera aguda, inhibidores no selectivos y selectivos de las COXs como el ácido flufenámico, flurbiprofeno o el NS-398 y mostraron una reducción significativa por liberación de renina, mientras que los inhibidores selectivos de COX-1 como el valeril salicilato

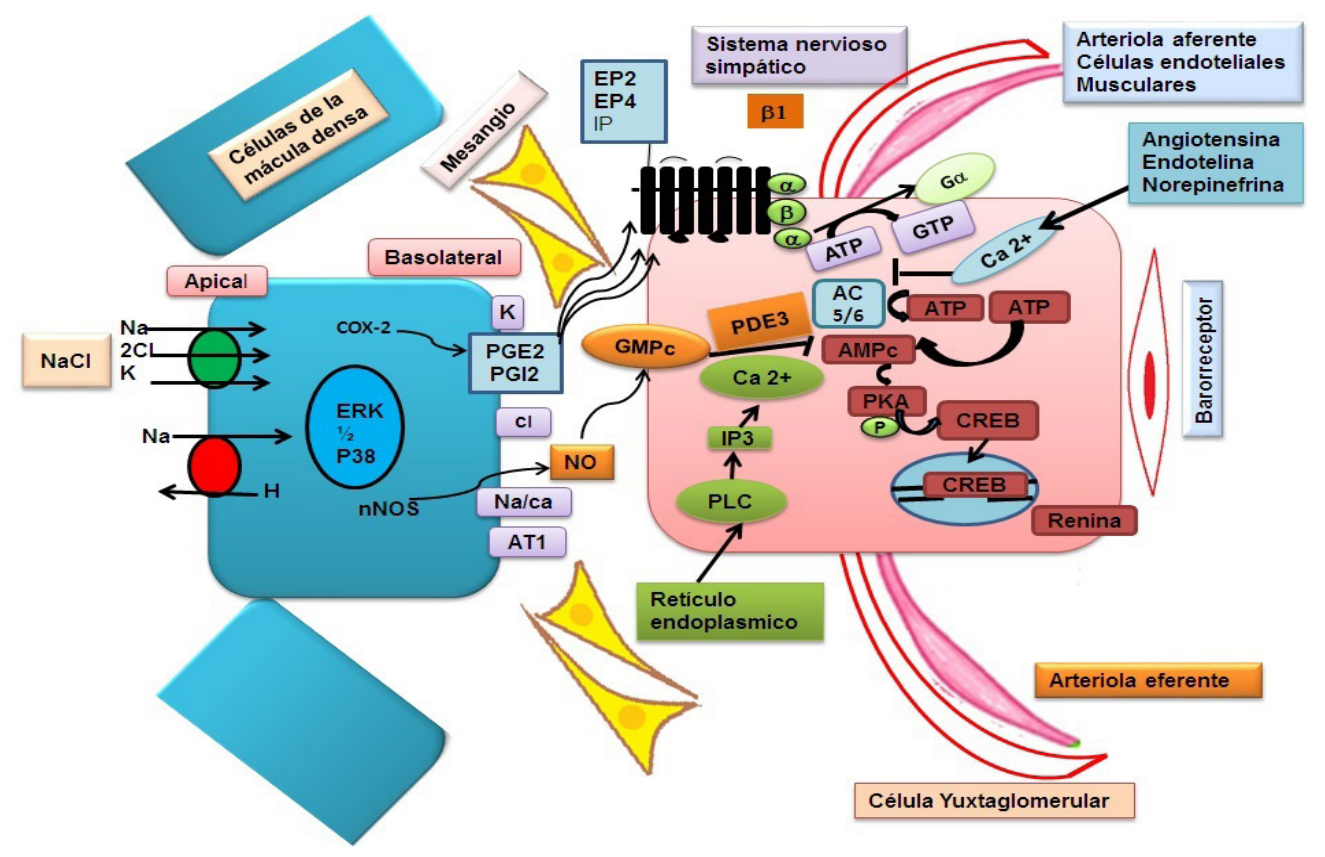

Figura 1. Aparato yuxtaglomerular: Existen tres vías principales que regulan la liberación de renina. el barorreceptor intrarrenal, la mácula densa y el sistema nervioso simpático y a través del AMP cíclico, inducen la liberación de renina de las células yuxtaglomerulares. Varios agonistas (por ejemplo,PGE2, norepinefrina, y PGI2), a través de sus receptores específicos, convergen en la activación de las proteínas Gso que activan a la adenilato ciclasa (AC5 y 6) e incrementa la concentración de monofosfato de adenosina cíclico (AMPc). La unión del AMPc a la subunidad reguladora de la proteína cinasa A (PKA) da lugar a la liberación de la subunidad catalítica; en el núcleo PKA fosforila a la proteína CREB que se une al elemento de respuesta al AMPc (CRE) ubicado en el DNA. La proteína CREB se une a la región del promotor del gen de renina para iniciar su transcripción. 


\section{Guzmán-Hernández et al.}

no mostraron efecto sobre la liberación de renina (29). In vivo, el aumento de la expresión de COX-2 y de la síntesis de prostaglandinas vasodilatadoras, en particular la $\mathrm{PGE}_{2}$ y la $\mathrm{PGI}_{2}$, estimulan la liberación de renina. Ratones con deleción de los receptores EP2 ${ }^{-/-}$, EP4 $4^{-/}$e IP ${ }^{-/}$, mostraron reducción significativa de la expresión de renina tras la administración de una dieta baja en $\mathrm{NaCl}$ durante una semana (30). Los receptores EP2, EP4e IP se acoplan a proteínas Gs y a la estimulación de la adenilato ciclasa 5 presente en las células secretoras de renina; la adenilato ciclasa cataliza la conversión de ATP en AMP cíclico (AMPc) que, a su vez, estimula la actividad de cinasas de proteínas como la PKA (31). El AMPc se une a dos subunidades regulatorias de la PKA para liberar dos subunidades catalíticas del complejo tetramérico inactivo de la PKA. Las subunidades catalíticas libres se traslocan al núcleo y fosforilan factores de transcripción como el elemento de respuesta al AMPc (CRE), el cual se une a la región consenso del gen de renina para iniciar su transcripción (32). El transcrito del gen de renina da lugar a la preprorrenina con un peso molecular de $48 \mathrm{kDa}$. La prorrenina enzimáticamente inactiva pasa al aparato de Golgi, para ser secretada de manera constitutiva, o para ser glicosilada y almacenada en las células yuxtaglomerulares. Dentro de las vesículas secretoras un propéptido de 43 aminoácidos, se escinde proteolíticamente para producir la renina cuyo peso molecular es de $41 \mathrm{kDa}$ (32) (Figura 1).

Liberación de renina de las celulas yuxtaglomerulares. Mientras que el AMPc es el principal estimulador de la liberación de renina, el $\mathrm{Ca}^{+}$se considera el principal inhibidor de su liberación. La Ang II, endotelina, vasopresina, y norepinefrina incrementan la entrada de $\mathrm{Ca}^{+}$ intracelular hacia la célula yuxtaglomerular causando su despolarización e inhibición de la liberación de renina (33). Mientras que la hiperpolarización de la membrana inducida por la activación del canal de $\mathrm{K}^{+}$o por la inhibición del canal de $\mathrm{Cl}^{-}$está acompañada por la liberación de renina (33). Una vez que se libera la renina de la célula yuxtaglomerular, el hígado libera el angiotensinógeno, sobre el cual actúa la renina para escindir un decapéptido de la región carboxilo terminal para formar la Ang I y esta, a través de la acción de la enzima convertidora de angiotensina (ECA), forma a la Ang II. Aunque hay dos subtipos principales de receptores: AT1 y AT2, el receptor AT1 es el principal responsable de las acciones hipertensinogénicas del SRA.

La Ang II, cuando se une al receptor AT1, ejerce un efecto de retroalimentación negativa sobre la expresión de la COX-2 a través de la activación de un pequeño grupo de cinasas dependientes de serina/treonina llamadas WNK's, que son proteínas atípicas con efectos pleiotrópicos y se localizan en el túbulo contorneado distal, túbulo conector y conducto colector. Estructuralmente las WNKs tienen un dominio cinasa, un dominio de autoinhibición, un sitio de autofosforilación y son abundantes en prolina, por lo que sus acciones se relacionan con la modulación del tráfico de proteínas desde o hacia la membrana plasmática y la fosforilación de proteínas de transporte. Para la fosforilación requieren de la participación de cinasas intermedias, llamadas SPAK (cinasa de serina y treonina rica en alanina relacionada con STE20/SPS1) (34).

En los seres humanos cuatro genes son los que codifican para las WNKs: WNK1, WNK2, WNK3 y WNK4. Debido a que la nefrona distal es sensible a varias hormonas, las WNKs son consideradas interruptores moleculares que modifican la actividad de diversos transportadores en función de las demandas fisiológicas. Por ejemplo, las WNKs regulan la actividad del cotrasportador NKCC2, el cotransportador clorosodio (NCC), y el canal epitelial de sodio (ENaC) (35).

\section{Revista Biomédica}




\section{Regulación de renina en hipertensión renovascular}

La Ang II,a través de su unión al receptor AT1, y de la activación de las WNK3 o WNK4 fosforilan y activan a SPAK, esta cinasa fosforila las treoninas 96, 101 y 111 contenidas en el cotransportador $\mathrm{NKCC} 2$, aumentando de esta manera su actividad, por lo que se incrementa la concentración de $\mathrm{NaCl}$ que llega hacia la $\mathrm{MD}$, inhibiendo de esta manera la expresión de la COX-2 (36). La disminución de la expresión de COX-2 reduce la síntesis de prostaglandinas E2 e I2, de manera que se reduce la liberación de renina y, así, la producción de angiotensina II.

\section{CONCLUSIÓN}

La hipertensión es una enfermedad muy común en la población con una alta tasa de complicaciones, su fisiopatología es compleja; en este trabajo, hemos abordado los principales mecanismos de regulación de la presión arterial, específicamente en la regulación de renina. En un modelo experimental de hipertensión renovascular, se observó que al disminuir la perfusión sanguínea renal se detecta una disminución del sodio luminal en la porción ascendente gruesa del asa de Henle que es sensado por el cotransportador NKCC2 de la mácula densa. Lo anterior, induce la expresión de la COX-2. COX-2 sintetiza $\mathrm{PGE}_{2}$ y $\mathrm{PGI}_{2}$ que estimulan la liberación de renina de las células yuxtaglomerulares y, con ello, la sobreactivación del sistema renina angiotensina; al aumentar la Ang II se eleva la presión arterial.

A su vez, la Ang II, a través de su unión al receptor AT1, y de la activación de las WNK3 o WNK4 fosforilan y activan a SPAK, esta cinasa fosforila las treoninas 96, 101 y 111 contenidas en el cotransportador $\mathrm{NKCC} 2$, lo que induce a que se incremente la concentración de $\mathrm{NaCl}$ en la MD, inhibiendo de esta manera la expresión de la COX-2 y de renina, y así, se reduce la síntesis de la hipertensinogénica angiotensina II.

\section{REFERENCIAS}

1. Lawes CM, Vander-Hoorn S, Rodgers A. International Society of Hypertension. Global burden of blood-pressure-related disease 2001. Lancet. 2008; 371(9623): 1513-1518.

2. Instituto Nacional de la Salud Pública. Secretaría de Salud. Encuesta Nacional de Salud y Nutrición. 2012. Resultados Nacionales. México: 113-116.

3. Stern RH. The new hypertension guidelines. J Clin Hypertens (Greenwich). 2013 Oct; 15(10): 748-51.

4. Coffman TM. The inextricable role of the kidney in hypertension. J Clin Invest. 2014 Jun; 124(6): 2341-7.

5. Ivy JR, Bailey MA. Pressure natriuresis and the renal control of arterial blood pressure. J Physiol. 2014 Sep 15; 592(Pt 18): 3955-67.

6. Chen D, Coffman TM. The kidney and hypertension: lessons from mouse models. Can J Cardiol. 2012 May; 28(3): 305-10

7. Powers B, Greene L, Balfe LM. Updates on the treatment of essential hypertension: a summary of AHRQ's comparative effectiveness review of angiotensin-converting enzyme inhibitors, angiotensin II receptor blockers, and direct renin inhibitors. J Manag Care Pharm. 2011 Oct; 17(8 Suppl): S1-14.

8. Navar LG, Kobori H, Prieto MC, GonzalezVillalobos RA. Intratubular renin-angiotensin system in hypertension. Hypertension. 2011 Mar; 57(3): 355-362.

9. Navar LG, Prieto MC, Satou R, Kobori H. Intrarenal angiotensin II and its contribution to the genesis of chronic hypertension. Curr Opin Pharmacol. 2011 Apr; 11(2): 180-6.

10. Matsusaka T, Niimura F, Shimizu A, Pastan I, Saito A, Kobori H, et al. Liver angiotensinogen is the primary source of renal angiotensin II. J Am Soc Nephrol. 2012 Jul; 23(7): 1181-9.

11. Navar LG, Harrison-Bernard LM, Wang CT, Cervenka L, Mitchell KD. Concentrations and actions of intraluminal angiotensin II. J Am Soc Nephrol. 1999 Jan; 10 (Supp111): S189-95.

12. Crowley SD, Zhang J, Herrera M, Griffiths R, Ruiz P, Coffman TM. Role of AT receptor-mediated salt retention in angiotensin II dependent hypertension. Am J Physiol Renal Physiol. 2011 Nov; 301(5): F1124-30.

13. Ohsawa M, Tamura K, Wakui H, Maeda A, Dejima T, Kanaoka T. Deletion of the angiotensin II type 1 receptor-associated protein enhances renal sodium reabsorption and exacerbates angiotensin II-mediated hypertension. Kidney Int. 2014 Sep; 86(3): 570-81. 
Guzmán-Hernández et al.

14. Prieto-Carrasquero MC, Botros FT, Kobori H, Navar LG. Collecting Duct Renin: A major player in Angiotensin II-dependent Hypertension. J Am Soc Hypertens. 2009 Mar-Apr; 3(2): 96-104.

15. Green T, Rodriguez J, Navar LG. Augmented cyclooxygenase-2 effects on renal function during varying states of angiotensin II. Am J Physiol Renal Physiol. 2010 Nov; 299(5): F954-62.

16. Korbecki J, Baranowska-Bosiacka I, Gutowska I, Chlubek D. Cyclooxygenase pathways. Acta Biochim Pol. 2014 Oct 23. En prensa

17. Castrop $H$ and Schieß IM. Physiology and pathophysiology of the renal Na-K-2Cl cotransporter (NKCC2). Am J Physiol Renal Physiol. 2014 Sep; 307: F991-F1002.

18. Edwards A, Castrop H, Laghmani K, Vallon V, and Layton AT. Effects of NKCC2 isoform regulation on $\mathrm{NaCl}$ transport in thick ascending limb and macula densa: a modeling study. Am J Physiol Renal Physiol. 2014 Jul; 307: F137-F146.

19. Mann B, Hartner A, Jensen BL, Hilgers KF, Höcherl K, Krämer BK, et al. Acute upregulation of COX-2 by renal artery stenosis. Am J Physiol Renal Physiol. 2001 Jan; 280(1): F119-25.

20. Boshra V, Hamid G, Nader M. Effect of celecoxib on the antihypertensive effect of losartan in a rat model of renovascular hypertension. Can J Physiol Pharmacol. 2011 Feb; 89: 102-107.

21. Kose F, Besen A, Paydas S, Balal M, Gonlusen G, Inal T, Dogan A, et al. Effects of selective Cox-2 inhibitor, rofecoxib, alone or combination with furosemide on renal functions and renal Cox2 expression in rats. Clin Exp Nephrol. 2010 Feb; 14(1): 22-7.

22. Cheng HF, Harris RC. Cyclooxygenase- 2 expression in cultured cortical thick ascending limb of Henle increases in response to decreased extracellular ionic content by both transcriptional and post-transcriptional mechanisms. Role of p38-mediated pathways. J Biol Chem. 2002 Sep; 277(47): 45638-43.

23. Zhang $Z$, Sheng $H$, Shao J, Beauchamp RD and DuBois RN. Posttranscriptional regulation of cyclooxygenase- 2 in rat intestinal epithelial cells. Proc Natl Acad Sci USA. 2005 Nov- Dec; 2(6): 523-30.

24. Yao J, Oite T, Kitamura M. Gap junctional intercellular communication in the juxtaglomerular apparatus. Am J Physiol Renal Physiol. 2009 May; 296(5): F939-46.

25. Prieto-Carrasquero MC, Botros FT, Pagan J, Kobori H, Seth DM, Casarini DE, et al. Collecting duct renin is upregulated in both kidneys of 2-kidney, 1-clip Goldblatt hypertensive rats. Hypertension. 2008 Jun; 51(6): 1590-6.

26. Castrop H, Höcherl K, Kurtz A, Schweda F, Todorov V, Wagner C. Physiology of kidney renin. Physiol Rev. 2010 Apr; 90(2): 607-73.

27. Gomez AR and Sequeira-Lopez ML. Who and where is the renal baroreceptor?: the connexin Hypothesis. Kidney Int. 2009 Mar; 75(5): 460-2.

28. Kim SM, Chen L, Faulhaber-Walter R, Oppermann M, Huang Y, Mizel D, et al. Regulation of renin secretion and expression in mice deficient in beta1and beta2-adrenergic receptors. Hypertension. 2007 Jul; 50: 103-9.

29. Kim SM, Briggs JP, Schnermann J. Convergence of major physiological stimuli for renin release on the Gs-alpha/cyclic adenosine monophosphate signaling pathway. Clin Exp Nephrol. 2012 Feb; 16(1): 17-24

30. Poschke A, Kern N, Maruyama T, Pavenstadt H, Narumiya S, Jensen B. The PGE2-EP4 receptor is necessary for stimulation of the renin-angiotensinaldosterone system in response to low dietary salt intake in vivo. Am J Physiol Renal Physiol. 2012 Nov; 303(10): 1435-1442.

31. Desch M, Harlander S, Neubauer B, Gerl M, Germain S, Castrop H, et al. cAMP target sequences enhCRE and CNRE sense low-salt intake to increase human renin gene expression in vivo. Pflugers Arch. 2011 May; 461 (5): 567-77.

32. Schweda F and Kurtz A. Regulation of Renin Release by Local and Systemic Factors. Rev Physiol Biochem Pharmacol. 2011 May; 161: 1-44.

33. Friis U, Kirsten Madsen, Stubbe J, Pernille B, .Hansen, Svenningsen \& Peter Skøtt $O$ et al Regulation of renin secretion by renal juxtaglomerular cells. Pflugers Arch - Eur J Physiol. 2013 Jan; 465: 25-37.

34. McCormick JA, Ellison DH. The WNKs: atypical protein kinases with pleiotropic actions. Physiol Rev. 2011 Jan; 91: 177-219.

35. Hoorn EJ, Nelson JH, McCormick JA, Ellison DH. The WNK kinase network regulating sodium, potassium, and blood pressure. J Am Soc Nephrol. 2011 Apr; 22(4): 605-14

36. Cristobal P, Pacheco-Alvarez $\mathbf{D}$, Richardson $\mathbf{C}$, Ring AM, Vazquez N, Rafiqi FH, et al. Angiotensin II signaling increases activity of the renal $\mathrm{Na}-\mathrm{Cl}$ cotransporter through a WNK4-SPAK-dependent pathway. Proc Natl Acad Sci USA. 2009 Mar; 1106(11): 4384-9. 